\title{
Symmetry breaking transition towards directional locomotion in Physarum microplasmodia
}

\author{
Shun Zhang ${ }^{1,2}$, Juan C. Lasheras ${ }^{1,3,4} \&$ and Juan C. del Álamo ${ }^{1,4}$ \\ ${ }^{1}$ Mechanical and Aerospace Engineering Department, University of California San \\ Diego \\ ${ }^{2}$ Cancer Institute, The Affiliated Hospital of Qingdao University \\ ${ }^{3}$ Department of Bioengineering, University of California San Diego \\ ${ }^{4}$ Institute for Engineering in Medicine, University of California San Diego \\ E-mail: shz019@ucsd.edu, jc@ucsd.edu
}

\begin{abstract}
True slime mold Physarum polycephalum has been widely used as a model organism to study flow-driven amoeboid locomotion as well as the dynamics of its complex mechanochemical self-oscillations. The aim of this work is to quantify the mechanical aspects of symmetry breaking and its transition into directional flow-driven amoeboid locomotion in small $(<\sim 200 \mu \mathrm{m})$ fragments of Physarum polycephalum. To this end, we combined measurements of traction stresses, fragment morphology, and ectoplasmic microrheology with experimental manipulations of cell-substrate adhesion, cortical strength and microplasmodium size. These measurements show that initiation of locomotion is accompanied by the symmetry breaking of traction stresses and the polarization of ectoplasmic mechanical properties, with the rear part of the microplasmodium becoming significantly stiffer after the onset of locomotion. Our experimental data suggests that the initiation of locomotion in Physarum could be analogous to an interfacial instability process and that microplasmodial size is a critical parameter governing the instability. Specifically, our results indicate that the instability driving the onset of locomotion is strengthened by substrate adhesiveness and weakened by cortical stiffness. Furthermore, the Fourier spectral analysis of morphology revealed lobe number $n=2$ as the consistent dominant mode number across various experimental manipulations, suggesting that the instability mechanism driving the onset of Physarum locomotion is robust with respect to changes in environmental conditions and microplasmodial properties.
\end{abstract}

Keywords: amoeboid motility, traction force microscopy, Physarum, mechano-chemical waves, symmetry breaking, locomotion, soft robotics. 


\section{Introduction}

Physarum polycephalum is an acellular slime mold long used as model organism of amoeboid motility [1,2], unconventional computing [3], and biomimetic design of soft robots [4]. In nature, Physarum forms branching tubular vein networks up to meters long, which contract rhythmically producing alternating flows of endoplasmic fluid [5]. Sub-millimeter fragments (i.e., microplasmodia) of Physarum or droplets of its endoplasmic fluid can be prepared in the laboratory by a variety of methods [6]. Microplasmodia smaller than a few hundred microns do not develop prominent veins and are composed of a submembranous gel-like ectoplasm (i.e., the cortex) layer in addition to the endoplasmic fluid. When seeded onto a substrate, initially round Physarum microplasmodia oscillate rhythmically and, in some cases, these fluctuations lead to the elongation of the microplasmodium and its directional locomotion [7]. This transition is generally viewed as a symmetry breaking process $[7,8]$ but our understanding about the process is still limited. There is data suggesting that only microplasmodia with size larger than $\sim 100 \mu \mathrm{m}$ can break symmetry [9]. However, the dependence of this critical lengthscale on the mechanical properties of the microplasmodium, or in environmental factors such as substrate adhesiveness, is not well understood. Furthermore, it is unclear how ectoplasm remodeling during symmetry breaking in turn affects the mechanical properties of the microplasmodium or its interactions with the substrate.

Takagi and Ueda experimentally documented the emergence of self-organized non-symmetric cell thickness waves in Physarum plasmodia and protoplasm droplets, including standing, traveling and rotational waves $[10,11]$. Their work motivated mathematical models of the self-organized waves that considered mechano-chemical feedback between ectoplasm contractions, the endoplasmic flow driven by these contractions, and the transport by the flow of the chemical signal that regulates contractility [12-14]. These models were able to reproduce the experimental observations and predicted two key parameters affecting the emergence the mechano-chemical waves - the drag coefficient between the endoplasm and the ectoplasm, and the Péclet number that quantifies the relative importance of advection versus diffusion in the transport of chemical signals. More recent models have incorporated membrane deformability and substrate adhesiveness to simulate microplasmodial motility during symmetry breaking [15] and directional locomotion [16]. However, these models fail to recapitulate directional locomotion from self-organized mechano-chemical waves - they either produce cell shape oscillations with zero net locomotion [15] or prescribe ad hoc traveling wave patterns of active contractility and/or substrate adhesiveness $[16,17]$.

The partial success of mathematical models of symmetry breaking in Physarum microplasmodia implies that crucial aspects of this process are still beyond grasp. This conceptual void is in large part due to a paucity of detailed experimental measurements of the mechanics of symmetry breaking, which has opened a gap between the ability to generate testable ideas by modeling and the ability to experimentally test them, and vice versa. For instance, while existing mathematical models can predict how 
changing specific parameters (e.g., substrate friction or ectoplasm shear modulus) affects detailed observable variables (e.g., membrane shape or substrate traction stress), the existing experimental data have been until recently mostly limited to measurements of cell thickness over time in round static microplasmodia. Experimental studies of Physarum microplasmodial locomotion have become increasingly common in recent years [16, 18-21], but symmetry breaking has comparatively received little attention.

The main objective of the present effort was to shed light into the mechanical aspects of symmetry breaking of Physarum microplasmodia, and the subsequent onset of directional locomotion. To this end, we combined measurements of microplasmodium shape and traction stresses with experimental manipulations of substrate adhesiveness, ectoplasm integrity, and microplasmodium size. The experimental data is analyzed to study how a biological system like Physarum, break symmetry from its initial stable status and subsequently transit towards directional locomotion, and how this process is affected by these aforementioned mechanical factors. Our measurements show the initiation of locomotion is accompanied by a polarity in cellular mechanical properties and exertion of traction stresses. In addition, our experimental data indicates that instability driving the onset of locomotion is strengthened by substrate adhesiveness and weakened by cortical stiffness. Finally, our result suggests that the initiation of locomotion in Physarum is analogous to an instability process, thus provides detailed quantitative data for developing and validating mathematical models.

\section{Methods}

\subsection{Preparation of Physarum Microplasmodia}

Physarum polycephalum microplasmodia were prepared on surface culture as previously described [6,7]. First-generation plasmodia were grown from sporae on $1 \%$ agar gel (Granulated; BD) using $150 \times 15-\mathrm{mm}$ culture plates (BD) and fed with oat flakes (QUAKER). The plates were kept in a dark humid environment at room temperature for approximately 1 week. Second-generation fragments approximately $1 \times 1 \mathrm{~mm}$ in size were harvested from the growing areas of the first-generation plasmodium and transferred to fresh agar plate, where they were kept overnight. Subsequently, microplasmodia with sizes ranging between $\sim 10 \mu \mathrm{m}$ and $\sim 100 \mu \mathrm{m}$ were excised from the growing tips of the second-generation fragments and placed over collagen coated polyacrylamide (PA) gels embedded with fluorescent microspheres. A 1-mm-thick cap made of a $1 \%$ agar gel was placed over the sample with the dual purpose of preventing the PA gel from drying out and gently flattening the microplasmodium to facilitate its visualization, as shown previously [16]. The samples were imaged under the microscope immediately after.

\subsection{Gel Fabrication}

Collagen-coated PA gel pads of $1.5 \mathrm{~mm}$ thickness were prepared for traction force microscopy as previously described [22], following established protocols [23]. Each 
PA gel consisted of a bottom layer without fluorescent beads and a thin (10 $\mu \mathrm{m})$ top layer containing $1 \mathrm{\mu m}$ fluorescent beads (FluoSperes; molecular probes) that were used as fiduciary markers to track substrate deformation. The gels were fabricated using $5 \%$ acrylamide and $0.3 \%$ bisacrylamide (Fisher BioReagents), resulting in a Young's modulus of $8.73 \mathrm{kPa}$ [24]. The Poisson's ratio of PA was measured to be 0.46 using elastographic traction force microscopy [25]. PA gels were activated with sulfo-SANPAH (Thermal Scientific) under UV light and coated with $0.15 \mathrm{mg} / \mathrm{ml}$ collagen I (Corning).

\subsection{Microscopy}

A Leica DMI 6000B inverted microscope mounted on an automated stage (ASI) and controlled by a PC running Micro-Manager software was used for image acquisition [26]. Three acquisition protocols were followed. Bright-field-only image sequences were recorded over 8 hours under $10 \mathrm{X}$ magnification at a $1 / 30 \mathrm{~Hz}$ rate. Traction force microscopy acquisitions were performed both in the bright field and the fluorescent field over 8 hours at a $1 / 300 \mathrm{~Hz}$ rate. Each fluorescent field image consisted of a 40plane three-dimensional z-stack with an inter-plane spacing of $\Delta z=1 \mu \mathrm{m}$. Particle tracking microrheology acquisitions were performed over 10 seconds under $63 \mathrm{X}$ with an oil-immersed lens at $200 \mathrm{~Hz}$ rate.

\subsection{D Traction Force Microscopy}

The contractile forces generated by Physarum microplasmodia induced threedimensional deformation of their substrate, which was determined by measuring the motion of the fluorescent beads embedded in the PA gel by correlation microscopy. Each instantaneous fluorescent image z-stack was compared with a reference z-stack recorded when the substrate was not deformed, i.e. after the microplasmodia moved out of the field of view. This comparison was carried out by dividing both images into 3D interrogation boxes and maximizing the cross-correlation between each interrogation box and the corresponding interrogation box of the reference image. The maximization was performed using constraints that enforced mechanical equilibrium of forces as previously described [27].

The measured deformation at the top of the substrate was used as boundary condition for the equation of mechanical equilibrium for the PA gel as previously described $[22,28]$. Using this solution, we computed the deformation field in the whole polyacrylamide substrate, as well as the traction stress vector $\vec{T}=\left(T_{x z}, T_{y z}, T_{z z}\right)$ on its top surface. For the sake of presentation, this vector was decomposed into the component parallel to the measurement plane (i.e., in plane), $\vec{T}_{t}=\left(T_{x z}, T_{y z}\right)$, and the out-of-plane component $T_{n}=T_{z z}$. The spatial resolution (i.e. the distance between adjacent measurement points) of the traction stress measurements is defined by $1 / 2$-size of the interrogation boxes according to the Nyquist criterion. In the present experiments, the resolution in the $x$ and $y$ directions was $6.5 \mu \mathrm{m}$ and $10 \mu \mathrm{m}$ respectively under $16 \mathrm{X}$ and 10X magnification, while it was $20 \mu \mathrm{m}$ in the $z$ direction. 


\subsection{Pharmacological Treatments}

In order to stabilize or destabilize the actin filaments of Physarum's ectoplasm, we treated specimens with phalloidin and latrunculin A respectively [29,30]. Phalloidin treatment was performed by injecting $1 \mathrm{mM}$ of Phalloidin (Sigma) into secondgeneration molds under a Nikon SMZ-10 microscope using a PM 1000 cell micro-injection system (MicroData Instrument, Inc). The injected amount (100 nl) was less than 1\% of the volume of the second-generation mold, as calculated by the measured diameter of the tubular structure of the mold. After waiting for 2 hours, multiple microplasmodia were excised from the marching end as described above. Latrunculin A treatment was performed by transferring microplasmodia to $5 \mu \mathrm{M}$ Latrunculin A (Cayman) solution for 10 minutes, then washing with Milli-Q water (Millipore).

\subsection{Modifications of Substrate Adhesiveness}

To decrease or increase the substrate adhesiveness we treated the PA gel and agarose cap with Pluronic F-127 or Poly-L-Lysine, respectively [31,32]. The treatment was performed by soaking the gels in a $0.2 \%$ Pluronic F-127 (Sigma) or a $2.5 \mathrm{mg} / \mathrm{ml}$ Poly-LLysine solution for 1 hour. Subsequently, the gels were washed thoroughly with Milli-Q water three times before seeding the Physarum microplasmodia.

\subsection{Morphological Analysis of Microplasmodia}

To quantify the time-dependent shapes of Physarum microplasmodia, the perimeter $\vec{X}_{e}(s)$ of each microplasmodium was automatically delineated from bright-field images as described previously [28], and its centroid position $\vec{X}_{c}$ was calculated (see Figure $1 \mathrm{~A}-\mathrm{C})$. Then the distance from the centroid to each point in the perimeter was measured and expressed as a function of the arclength distance along the contour, i.e. $r(s)=\left|\vec{X}_{e}(s)-\vec{X}_{c}\right|$ such that $L=\oint d s$ is the perimeter length. The resulting function is periodic (i.e. $r(s)=r(s+L)$ ) and its number of peaks provides information about the number of lobes in the microplasmodium perimeter (see Figure 1D-F). Fourier spectral analysis of $r(s)=\sum_{n} R_{n} \exp (2 \pi i n s / L)$ was performed to identify how much each lobe number $n$ contributed to departures from circular shape. To this end, we compared $\left|R_{n}\right|, n>0$ with the average radius $R_{0}=L^{-1} \oint r(s) d s$, see Figure $\left.1 \mathrm{G}-\mathrm{I}\right)$.

\subsection{Directional Particle-tracking Microrheology (DPTM)}

Carboxylate modified red latex beads with $0.2 \mu \mathrm{m}$ nominal diameter (Fluospheres, Invitrogen, Carlsbad CA) were diluted with Milli-Q water in 1:1000 ratio. The solution was then injected into branched second-generation fragments (as described in Section 2.1) under a Nikon SMZ-10 microscope, using a PM 1000 cell micro-injection system (MicroData Instrument, Inc). The beads were dispersed by shuttle streaming and were evenly distributed across the fragments after 6 hours of injection. At this point, microplasmodia were excised and prepared as described above (Figure 2 A1). 

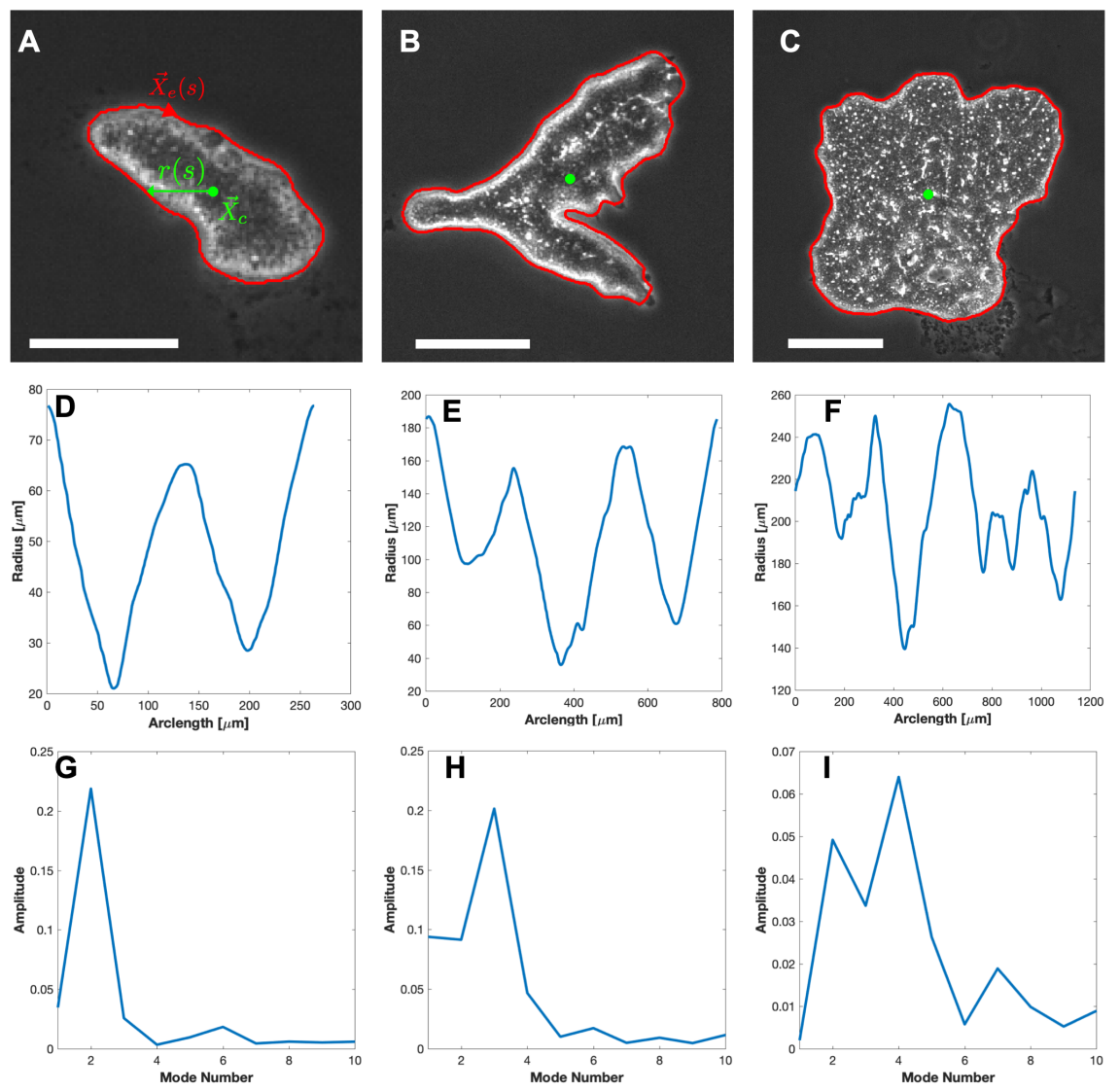

Figure 1: Shape analysis. (A,B,C) Snapshots of bright field images of Physarum microplasmodia with different morphologies. The red curve is detected outline of the microplasmodium and the green dot is its centroid. (D, E,F) Radial distance from the centroid to each point on the microplasmodium's outline, $r(s)$, vs. distance along the outline perimeter, s. $\quad(\mathbf{G}, \mathbf{H}, \mathbf{I})$ Normalized amplitude $\left|R_{n}\right| / R_{0}$ of the shape modes derived from the Fourier analysis of $r(s)$ vs. mode number $n$.

The motion of the beads embedded in microplasmodia was under the microscope as described above (Figure 2 A2). The centers of the fluorescent beads were tracked using previously described in-house algorithms [33]. The $2 \times 2$ mean square displacement (MSD) tensor of the beads, $\left\langle\Delta x^{2}\right\rangle_{i, j}(\tau)=\left\langle\left[x_{i}(t+\tau)-x_{i}(t)\right]\left[x_{j}(t+\tau)-x_{j}(t)\right]\right\rangle$ was calculated from their tracked trajectories, and their eigenvalues $\left\langle\Delta x^{2}\right\rangle_{\|}$and $\left\langle\Delta x^{2}\right\rangle_{\perp}$ along the principal directions of maximal and minimal mobility were calculated as previously described [34].

Beads were categorized in three groups according to the shape of their corresponding $\left\langle\Delta x^{2}\right\rangle_{\|}(\tau)$ and $\left\langle\Delta x^{2}\right\rangle_{\perp}(\tau)$ curves. About $10 \%$ of the beads showed isotropic diffusive behavior $\left\langle\Delta x^{2}\right\rangle_{\|} \approx\left\langle\Delta x^{2}\right\rangle_{\perp} \sim \tau$ at short times that saturated to a constant value $l^{2} \sim 1 \mu m^{2}$, suggesting that these beads were trapped in vesicles or membrane invaginations (Figure $2 \mathrm{C} 1, \mathrm{D} 1$ ). Another $40 \%$ of the beads exhibited ballistic motion with $\left\langle\Delta x^{2}\right\rangle_{\|} \sim\left\langle\Delta x^{2}\right\rangle_{\perp} \sim v^{2} \tau^{2}$, suggesting that they were being carried by endoplasmic 

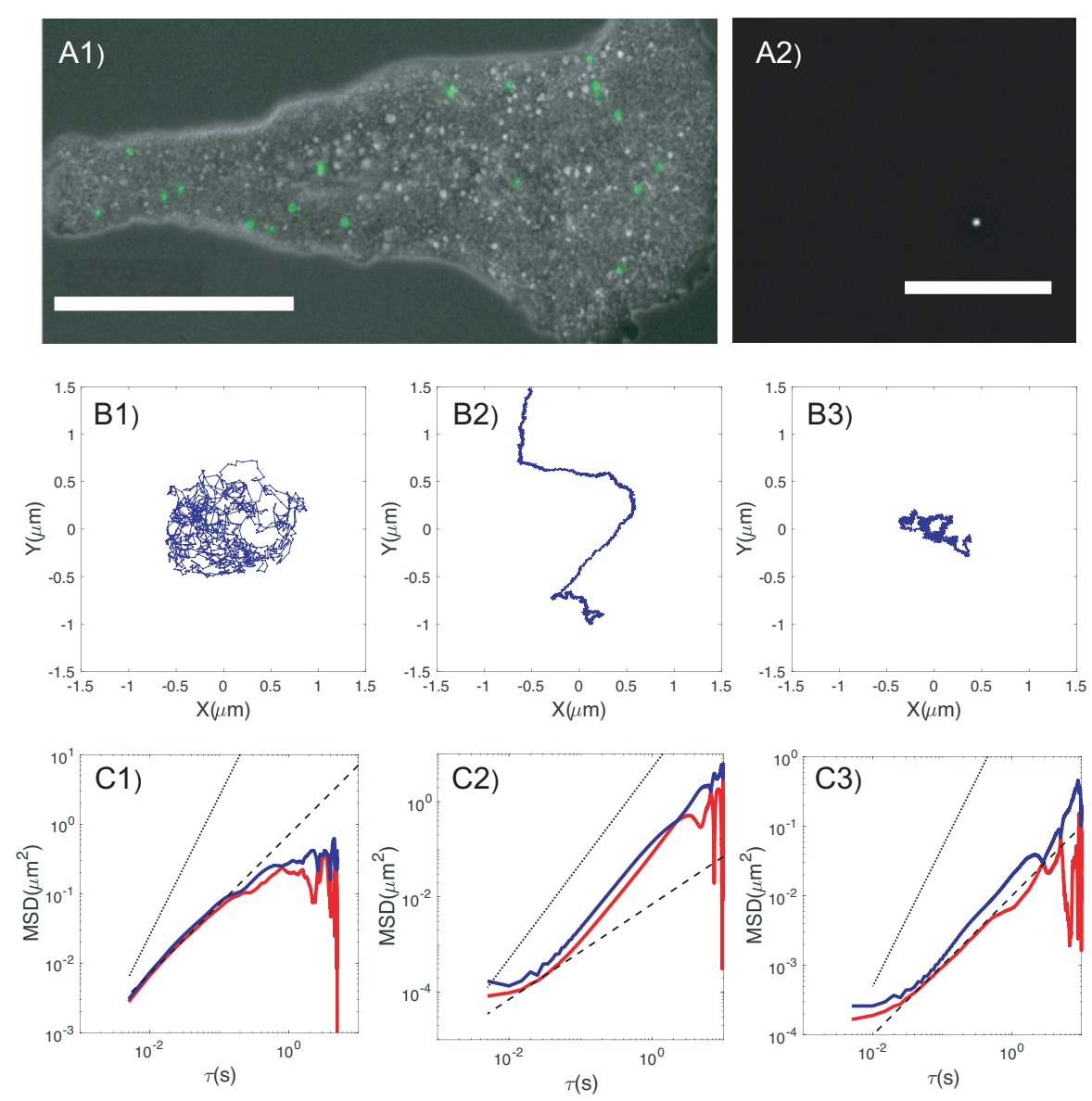

Figure 2: DPTM measurements. (A1) Distribution of embedded microparticles in microplasmodial fragment. Scale bar $=100 \mu \mathrm{m}$. (A2) Representative fluorescence image of microparticle acquired in experiment. Scale bar $=20 \mu \mathrm{m}$. (B1,B2,B3) Trajectories of microparticles. (C1,C2,C3) Corresponding MSD in the principal directions of maximal (II) and minimal $(\perp)$ motility for trajectories in (B1-B3). - $-\left\langle x^{2}\right\rangle_{\|} ;-,\left\langle x^{2}\right\rangle_{\perp} \cdot---$, $\left\langle x^{2}\right\rangle \sim \tau ; \cdots \cdots \cdots,\left\langle x^{2}\right\rangle \sim \tau^{2}$

shuttle flow (Figure 2C2, D2). The typical values of $v$ estimated from particle MSDs, $v \sim 0.5 \mu \mathrm{m} / \mathrm{s}$, are in good agreement with previously reported values of endoplasmic velocities in Physarum microplasmodia [7,16]. The remaining $\approx 50 \%$ of the tracked beads were diffusive or sub-diffusive in the $\perp$ direction (Figure 2C3, D3), suggesting that they were embedded in the F-actin cytoskeleton of the microplasmodium. The II direction of the MSDs for these beads was slightly superdiffusive as a consequence of the slow net directional flow of the ectoplasm, which we previously reported [20]. Thus, we used the measured $\left\langle\Delta x^{2}\right\rangle_{\perp}$ for these beads to estimate the shear modulus of the cytoskeleton using the generalized Stokes-Einstein (GSER) relationship [35,36]. Given that the GSER assumes thermodynamical equilibrium but the MSDs of these beads at timescales longer than $\tau \approx 0.1 \mathrm{~s}$ are possibly affected by active out-of-equilibrium processes (e.g. contractility of the cytoskeleton) [37,38], we only considered MSD data with $\tau<0.1$ s in our (GSER) analysis. 

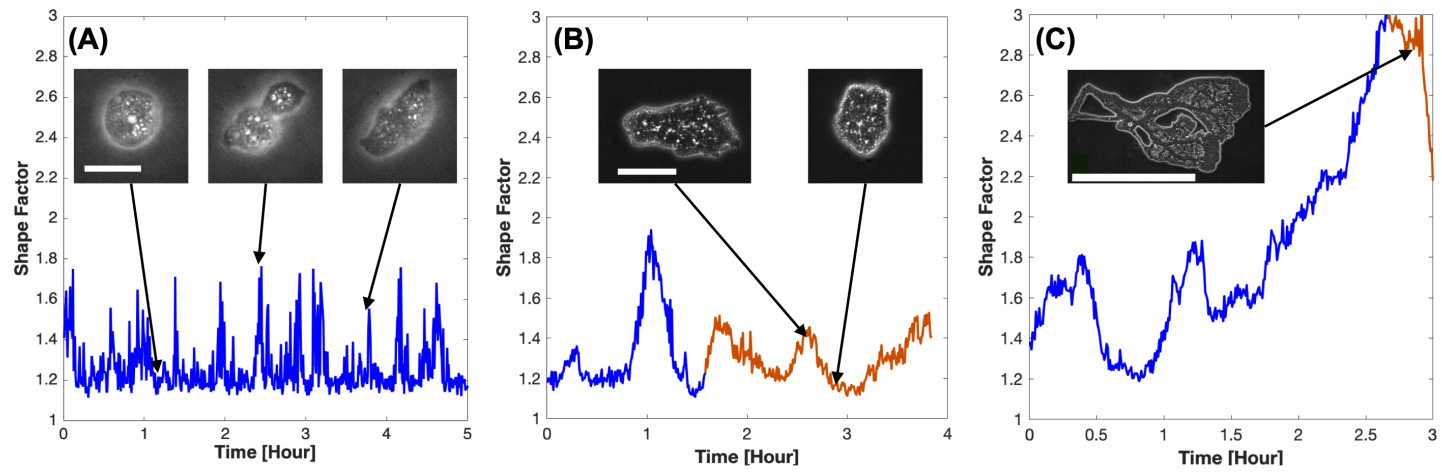

Figure 3: Time histories of the shape factor, $S F=\frac{L^{2}}{4 \pi R_{0}^{2}}$, for three Physarum plasmodia with different sizes and behavior. Blue color indicates the time interval before the plasmodia initiate locomotion, while orange indicates directional locomotion. The insets show bright field snapshots for the plasmodium corresponding to each $S F$ plot. (A) Small microplasmodium (scale bar $=20 \mu \mathrm{m})$ that does not initiate locomotion despite undergoing marked shape fluctuations. (B) Larger microplasmodium (scale bar $=100 \mu \mathrm{m}$ ) that initiates locomotion approximately 90 minutes after seeding on the substrate. (C) Mesoplasmodium (scale bar = $1 \mathrm{~mm}$ ) that initiates locomotion approximately 150 minutes after seeding on the substrate.

\section{Results and Discussion}

\subsection{Plasmodial shape dynamics upon substrate seeding}

The size (e.g., average radius $R_{0}$, see section 2.7) of Physarum plasmodia can be varied across several orders of magnitudes by their method of preparation, resulting in specimens with markedly different morphologies and behaviors. Figure 3 illustrates this dependence in the range $10 \mu \mathrm{m} \lesssim R_{0} \lesssim 1 \mathrm{~mm}$. Upon seeding onto the substrate, Physarum fragments with $R_{0} \lesssim 30 \mu \mathrm{m}$ remained mostly rounded and were unable to initiate directional locomotion despite undergoing considerable shape fluctuations (Figure 3A). Fragments of size $R_{0} \sim 100 \mu \mathrm{m}$ underwent a $\sim 2$-hour-long phase of oscillations about a circular shape, followed by a rapid transient in which they adopted a tadpole-like shape and began crawling directionally. Finally, Physarum fragments larger than a few hundred microns were also able to crawl directionally but developed a more complex morphology, including a posterior branched vein network (Figure 3C). These fragments are usually called mesoplasmodia as they share some of the key features of both microplasmodia and the larger arborescent plasmodia found in nature. The emergence of their tubular morphology and its effect on locomotion have been previously studied [21,39], and were beyond the scope of the present study. Here we focus on microplasmodia with homogeneous endoplasmic and ectoplasmic phases (i.e., without tubular networks) in the range of characteristic radius $10 \mu \mathrm{m} \lesssim R_{0} \lesssim 200 \mu \mathrm{m}$ where the transition to locomotion takes place. 


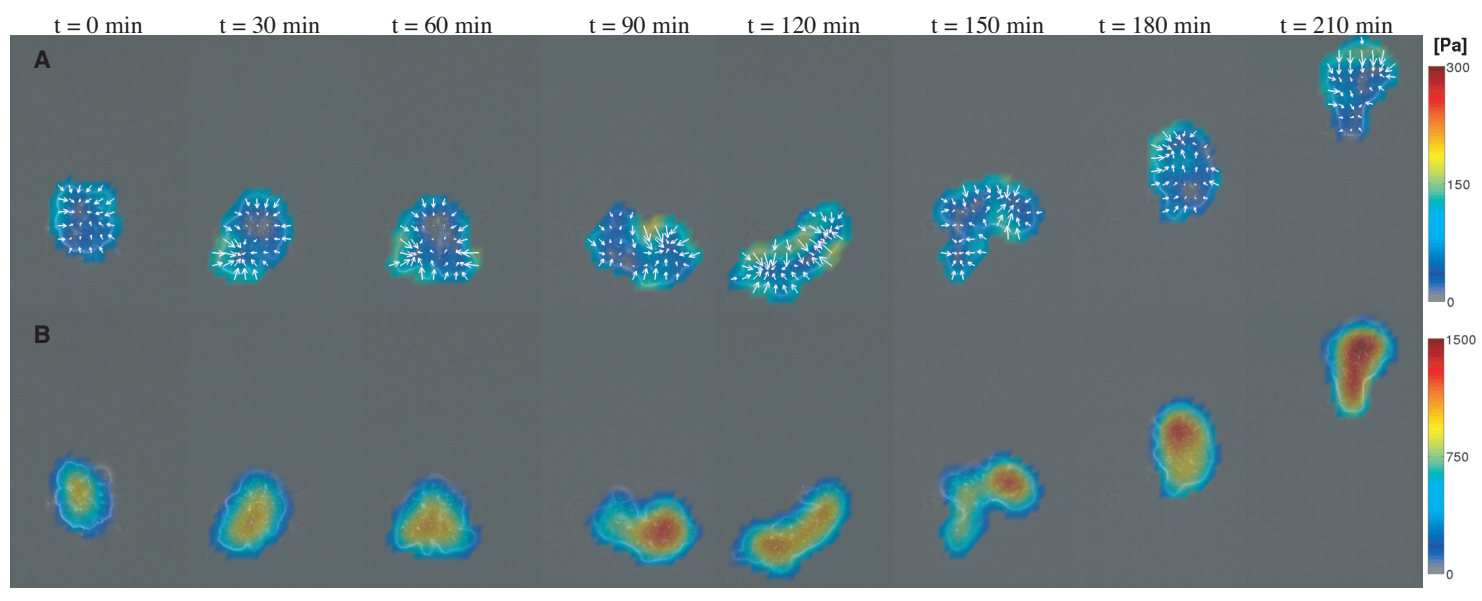

Figure 4: Time evolution of the 3-D traction stresses exerted by a Physarum microplasmodium on its substrate during the initiation of locomotion. (a) In-plane traction stress vector $\vec{T}_{t}=\left(T_{x z}, T_{y z}\right) ;(b)$ Out-of-plane traction stress $T_{n}=T_{z z}$.

\subsection{The onset of locomotion is accompanied by a symmetry breaking in traction stresses and ectoplasmic mechanical properties}

To better understand the mechanics of the onset of locomotion of Physarum microplasmodia, we measured the 3-D traction stresses they exerted on their substrate both in-plane $\vec{T}_{t}=\left(T_{x z}, T_{y z}\right)$ and out-of-plane $T_{n}=T_{z z}$. Figure 4 shows a representative example of the time evolution of these stresses during the symmetry breaking process. Initially $(t \lesssim 60 \mathrm{~min})$, the microplasmodium contracted and relaxed rhythmically while keeping a round shape, similar to the behavior shown in Figure 3. During this phase, it created inward contractile $\vec{T}_{t}$ distributed almost uniformly along its body's contour (Figure $4 a$ ). At the same time, it exerted intense downward compressive $T_{n}$ that peaked under the center of the macroplasmodial body (Figure $4 b$ ). This traction stress pattern is similar to that created by the surface tension and associated Laplace pressure of a vesicle deposited on a soft substrate [40], suggesting that the dynamics of cortical tension could govern the initiation of locomotion in Physarum.

As the microplasmodium adopted a more elongated shape and started translocating slowly $(60 \mathrm{~min} \lesssim t \lesssim 150 \mathrm{~min}$ ), the traction stresses lost their initial circular symmetry; however, they had not developed a clear front-back polarity yet. Once the microplasmodium adopted a tadpole-like shape and began directional locomotion (i.e., $t \gtrsim 150 \mathrm{~min}$ ), the traction stresses became more polarized. In particular, the frontal value of $T_{n}$ became much stronger than its posterior value (Figure $4 b$ ). This gradient in out-of-plane stresses suggests that the cortex/membrane structure of the microplasmodium bears higher tension in its rear part than in its front. The higher posterior tension would permit a higher pressure difference between the interior and the exterior of the microplasmodium, resulting in lower substrate compression under the plasmodium's tail. The alternative would be that intracellular pressure was continuously 
higher at the front of the plasmodium, which would be hard to reconcile with the shuttle streaming flows of alternating directions observed in these microplasmodia [7, 16, 19].

It is possible that the polarization of microplasmodial morphology contributes to polarization in tension, as a tadpole-like shape produces higher membrane curvature at the rear of the microplasmodium. In addition, symmetry breaking may cause longterm polarization in the composition and mechanical properties of the cytoskeleton. The density of myosin II heads in the F-actin cortex is known to increase towards the rear of the microplasmodium [41], and this protein is known to act as a major crosslinker of actin filaments [42]. Moreover, the frontal F-actin cortex undergoes significant remodeling including disassembly and reassembly coinciding with the cyclic contraction and relaxation of the microplasmodium, whereas the posterior cortex has a less dynamic structure [43]. To evaluate the effects of symmetry breaking on the mechanical properties of Physarum fragments, we used directional particle tracking microrheology (DPTM) [33-36] to measure the ectoplasmic shear modulus at different locations of the microplasmodium, both before and after the onset of locomotion. In DPTM (see $\S 2.8$ for details), the trajectories of beads embedded in the microplasmodium are tracked with high temporal resolution and their $2 \times 2$ mean squared displacement (MSD) tensor, $\left\langle\Delta x^{2}\right\rangle_{i, j}(\tau)=\left\langle\left[x_{i}(t+\tau)-x_{i}(t)\right]\left[x_{j}(t+\tau)-x_{j}(t)\right]\right\rangle$, is determined. Based on the shape of the MSDs, we identified particles embedded inside the ectoplasm and discarded particles embedded in vesicles or in the streaming endoplasm. The slow net motion of the ectoplasm was dedrifted by analyzing the smallest eigenvalue of the MSD tensor.

We first measured the MSDs vs. $\tau$ for ectoplasm-embedded particles at frontal and rear locations of the microplasmodia (Figure $5 a$ ). For $\tau \lesssim 0.1 s$, the MSDs at the rear fell by approximately two-fold, whereas the MSDs at the front did not appreciably change. In contrast, for $\tau \gtrsim 0.1 s$, the MSDs did not change after symmetry breaking and remained independent of particle position. The $\tau$-dependence of these results can be interpreted considering that, for $\tau \lesssim 0.1 s$, the random motion of particles embedded inside an actomyosin network is driven by thermal fluctuations so that their MSDs reflect the microrheological properties of the network. On the other hand, for $\tau \gtrsim 0.1 s$ the particle motions reflect network fluctuations driven by motor proteins and the MSDs measure the intensity of this active process $[37,38]$. Thus, our results suggest that symmetry breaking causes a change in the mechanical properties of the ectoplasm at the rear part of the microplasmodium, while its contractile activity seems to remain unchanged.

To quantify the changes in ectoplasm microrheological properties after symmetry breaking, we applied the Generalized Stokes-Einstein Relation (GSER, see $\S 2.8$ for details) to the measured MSDs in the range $0.005 s \leq \tau \leq 0.1 s[35,36]$. This analysis provided loss (viscous) and storage (elastic) shear moduli as a function of frequency $\omega$, $G^{\prime \prime}(\omega)$ and $G^{\prime}(\omega)$ respectively. Figure $5(b)$ shows values of $G^{\prime}$ and $G^{\prime \prime}$ for $\omega=100 \mathrm{~s}^{-1}$, indicating that $G^{\prime}$ at the rear of the microplasmodium increases dramatically after symmetry breaking while $G^{\prime \prime}$ experiences a moderate increase. Of note, these changes also imply a transition in the material response of the rear ectoplasm from mostly viscous 

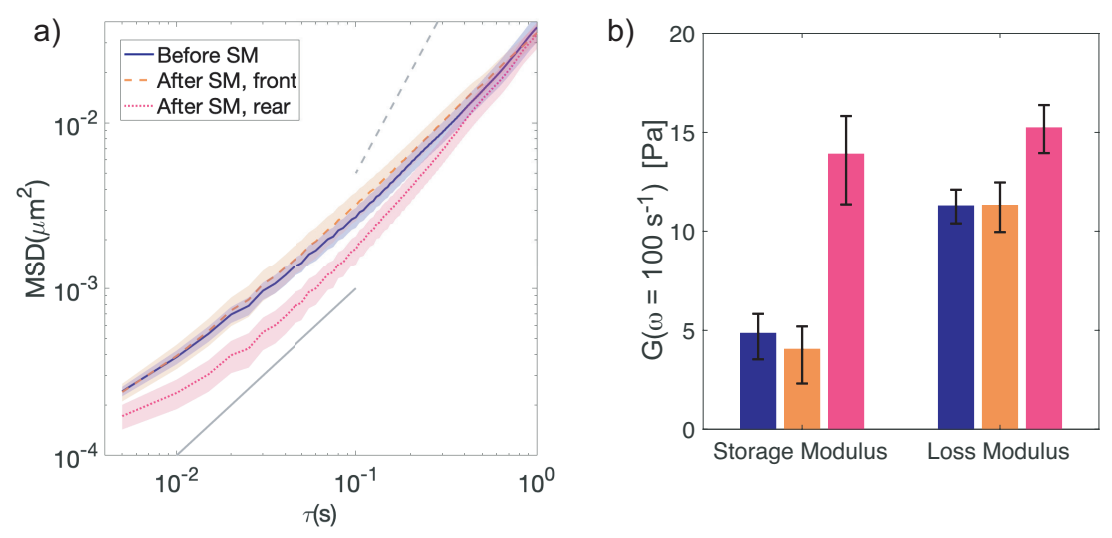

Figure 5: Quantification of the microrheological properties of the ectoplasm in Physarum microplasmodia. (a) MSD of ectoplasm-embedded particles in the principal direction of minimum mobility $\left(\left\langle x^{2}\right\rangle_{\perp}\right)$. The data come from Physarum fragments before initiation of locomotion $(\mathrm{N}=42,-)$, as well as in the head $(\mathrm{N}=25,--)$ ) and tail $(\mathrm{N}=14$, $\cdots \cdots$ ) regions of fragments after the onset of directional locomotion. The regions between the average MSD \pm one standard error are shaded in corresponding colors. $\cdots \cdots \cdots,\left\langle x^{2}\right\rangle_{\perp} \sim \tau$; ,$---\left\langle x^{2}\right\rangle_{\perp} \sim \tau^{2}$; (b) Bar plots of storage and loss moduli at $100 \mathrm{~Hz}$ calculated from the MSD data in panel $(a)$. Error bars correspond to one standard error. The colorscheme is the same as in panel $(a)$.

$\left(G^{\prime \prime}>G^{\prime}\right)$ before symmetry breaking to viscoelastic $\left(G^{\prime \prime} \approx G^{\prime}\right)$ afterward. In contrast, the two components of the shear modulus remained almost constant at the front of the microplasmodium, and their response continued to be viscosity-dominated.

It has long been speculated that the polarization of cortex stiffness may provide a mechanism to select synchronous mechano-chemical wave patterns that facilitate directional locomotion $[10,11]$. This idea is based on the observation that protoplasmic droplets with uniform cortical structure often bear rich spatio-temporal mechanochemical dynamics including chaotic patterns [11], whereas tadpole-shaped locomoting microplasmodia very rarely do so [20]. In fact, prescribing a softer front and a stiffer rear in mathematical models of Physarum motility facilitates reproducing experimentally observed behaviors, including directional locomotion $[12,16]$. The posterior network of thick-walled veins that develops in mesoplasmodia and larger plasmodia structurally recapitulates a front-back stiffness gradient. Furthermore, it has been shown to act as a low-pass filter that selects organized low-frequency oscillations, facilitating efficient locomotion [21]. As mentioned above, tadpole-shaped microplasmodia also develop a front-back gradient in cortical integrity even if they do not form posterior veins [41]. Our microrheological measurements provide direct evidence that the posterior part of Physarum microplasmodia undergo stiffening during the symmetry breaking process that precedes the onset of directional locomotion. 


\subsection{The onset of locomotion is a transition governed by microplasmodium size}

To systematically study the role of microplasmodium size in the initiation of locomotion, we tracked the time-dependent shape and position of $N=61$ microplasmodia of different sizes and categorized them as locomoting or non-locomoting according to their centroid displacement $\Delta X_{c}$ relative to time-averaged radius $\left\langle R_{0}\right\rangle_{T}$ over a period $T=6$ hours, i.e.

$$
\max \left(\left|\Delta X_{c}\right|, 0 \leq t \leq T\right)>\left\langle R_{0}\right\rangle_{T} \quad \longrightarrow \quad \text { locomoting. }
$$

For those microplasmodia that transitioned to directional locomotion, the instant $\tau_{\text {loc }}$ at which $\left|\Delta X_{c}\right|\left(\tau_{\text {loc }}\right)=\left\langle R_{0}\right\rangle_{\tau_{\text {loc }}}$ was defined as the time required for initiation of locomotion.

We found that the rate of locomoting microplasmodia increased with $\left\langle R_{0}\right\rangle_{T}$ and reached $100 \%$ for $\left\langle R_{0}\right\rangle_{T} \approx 80 \mu \mathrm{m}$ (Figure $6 a$ ). This result agrees with Koya and Ueda [9], who studied the emergence of shuttle streaming in microplasmodia formed by coalescence of tiny fragments, each containing approximately eight nuclei, and reported that both the morphological complexity and endoplasmic streaming velocity of the fused microplasmodia experienced a sharp increase beyond a critical size of 20 fragments. Altogether, the available experimental evidence suggests that the initiation of locomotion in Physarum is analogous to an instability process and that microplasmodial size is a critical parameter governing the instability. Consistent with this idea, linear stability analyses of mathematical models of Physarum as poroelastic mechano-chemical droplets yield growth rates that decrease with decreasing perturbation wavelength $[14,44]$.

\subsection{The onset of directional locomotion is governed by ectoplasm integrity and substrate adhesiveness}

Next, we studied how the onset of locomotion is affected by the mechanical properties of the microplasmodium and its environment. We first investigated the effect of ectoplasm integrity by pharmacological manipulation of the F-actin cytoskeleton. Physarum has a thick cortical ectoplasm composed by F-actin that surrounds the interior of its membrane and is crucial for the generation of the forces and shape changes required for locomotion [45]. To strengthen the ectoplasm, we treated microplasmodia with Phalloidin, which binds to actin filaments and stabilizes them by inhibiting their depolymerization, thereby promoting cortical thickening [46]. To disrupt the ectoplasm, we treated microplasmodia with Latrunculin A (LatA), which prevents actin monomers from polymerizing and has been shown to weaken the cortex in Physarum [47]. For each type of treatment, we tracked a large number of microplasmodia for $T=6$ hours and determined the rate of locomoting specimens as a function of their size, similar to Figure $6(a)$. These experiments revealed that phalloidin-treated microplasmodia of a given size are less prone to initiating locomotion than LatA-treated and control microplasmodia of the same size (Figure 6b). Similar to the control case, Phalloidintreated microplasmodia transitioned from non-locomoting to locomoting as their size 

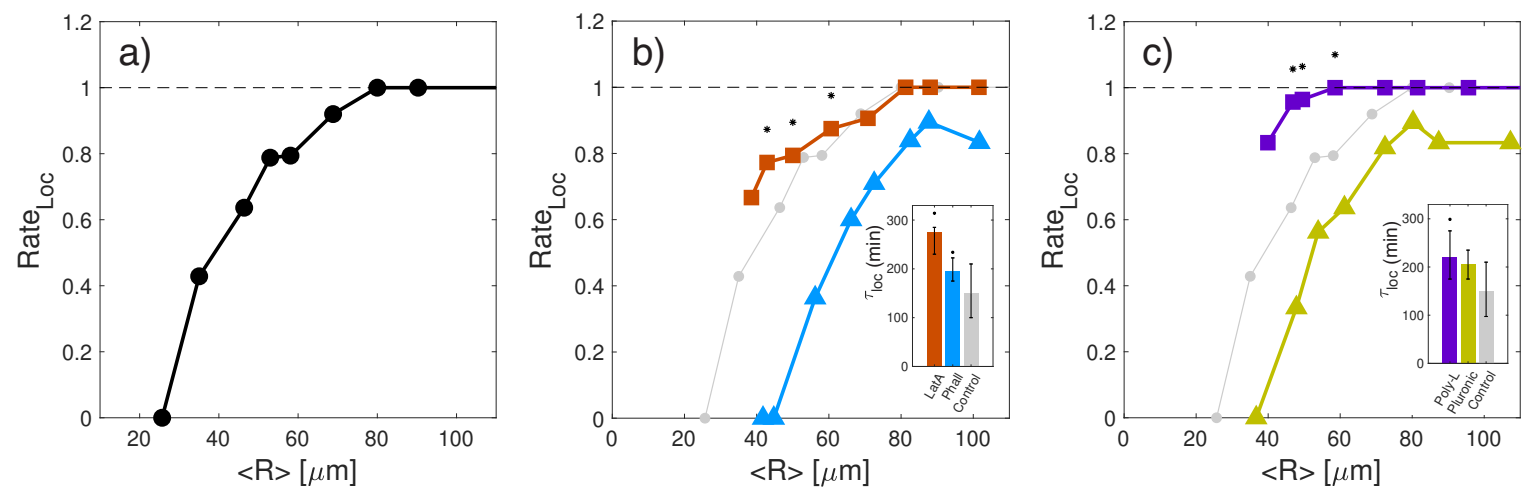

Figure 6: Size, cortical integrity and substrate adhesiveness govern the onset of locomotion. (a) Fraction of Physarum microplasmodia that initiate directional locomotion during the $T=6$ hrs ensuing seeding on the substrate, Rate Loc , vs. time-averaged microplasmodium size $\left\langle R_{0}\right\rangle$ $(N=61)$. (b) Rate $_{l o c}$ vs. $\left\langle R_{0}\right\rangle$ for microplasmodia treated with latrunculin A (orange squares, $N=70$ ) and phalloidin (blue triangles, $N=48$ ). The control condition from panel $(a)$ is included with light gray circles for reference. The asterisks denote statistically significant differences between the latrunculin A and phalloidin conditions $(p<0.05$ according to Fisher's exact test for categorical variables). (c) Rate loc $_{\text {vs. }}\left\langle R_{0}\right\rangle$ for microplasmodia seeded on substrates coated with poly-L-lysine (purple squares, $N=66$ ) and pluronic (yellow triangles, $N=39$ ). The control condition from panel $(a)$ is included with light gray circles for reference. The asterisks denote statistically significant differences between the poly-L-lysine and pluronic conditions ( $p<0.05$ according to Fisher's exact test for categorical variables). The inset barplots in $\mathbf{b}-\mathbf{c}$ indicate the median time for initiation of locomotion in each case (error bars denote $95 \%$ confidence intervals), $\tau_{l o c}$. The asterisks denote statistically significant differences with respect to the control case $(p<0.05$ according to Wilcoxon ranksum test).

increased, but this transition shifted by $\approx 20 \mu \mathrm{m}$.

If one idealizes the microplasmodia as a two-phase active poroelastic material, the observed behavior could be explained by the fact that for the same level of contractile force, a stiffer gel phase (ectoplasm) would tend to deform less and squeeze less sol phase (endoplasm). Consistent with this idea and our experimental results, the most unstable wavelength in linear stability analyses of Physarum droplets increases with gel phase stiffness [44]. One could also consider that the F-actin network in Phalloidin-treated microplasmodia likely has a smaller pore size, which would cause an increase in the drag coefficient between the gel and sol phases. This effect would damp the growth rate of perturbations, but it would also decrease the wavelength of the most unstable perturbations [14], which is at odds with our experimental data showing that the critical size for symmetry breaking increased with phalloidin treatment (Figure 6b).

An alternate explanation for our experimental observations could be that the interfacial tension and bending stiffness of the cortex/membrane system hinder the initiation of locomotion by damping shape perturbations that increase local cortex curvature (e.g., pseudopod protrusions). In consonance with Figure $6(a)-(b)$, this mechanism would make microplasmodia of smaller size less likely to initiate directional 
locomotion. Also in consonance with that figure, this mechanism would make microplasmodia with stronger (weaker) cortices - i.e., Phalloidin-treated (LatA-treated) - more (less) stable than controls. Of note, in smaller Dictyostelium amoeboid cells, genetic manipulation of cortex-specific proteins has shown that strengthening cortical strength limits cell migration speed [40]. We are not aware of mathematical models that specifically investigate the effect of cortical integrity in the initiation of Physarum migration. However, researchers have explored the general idea of interfacial instabilities in single-phase active fluids [48,49], offering insight that is at least qualitatively applicable to our experiments. These studies suggest that initially-round cell fragments can become unstable if their size exceeds a critical radius $R_{c r i t}$ and that cortical tension and bending stiffness can shift the instability to larger radii. Both predictions are in qualitative agreement with our experimental data (Figure $6 a-b$ ).

An additional qualitative prediction of interfacial instability models is that increasing substrate adhesiveness should contribute to destabilizing microplasmodial shape [48] - similar to increasing the capillary number of the interface of a passive droplet [50]. To test this hypothesis, we experimentally modulated the effect of substrate adhesiveness by performing experiments on substrates coated with either Pluronic F-127 or Poly-L-Lysine. Pluronic F-127 is a bio-compatible PEG-based triblock copolymer surfactant that reduces specific and non-specific adhesion [31]. Poly-L-Lysine is a positively charged polymer that increases substrate adhesiveness by electrostatic interactions with the negatively charged cell membrane [32,51]. Our experimental results (Figure 6c) indicate that microplasmodia of similar size are more likely to initiate locomotion on more adhesive substrates, whereas the opposite is true for less

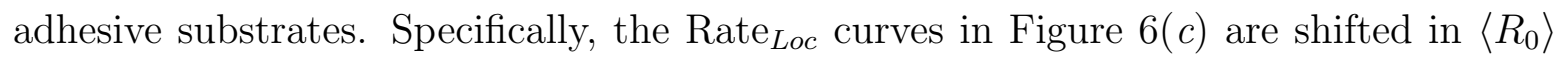
by approximately $-20 \mu \mathrm{m}$ and $+15 \mu \mathrm{m}$ for Poly-L-Lysine and Pluronic F-127 treated substrates, respectively.

While the measured Rate $_{L o c}$ curves are in good qualitative agreement with previous model predictions, the measured times for initiation of locomotion $\left(\tau_{l o c}\right.$, see insets in Figure $6 b-c$ ) call for caution when interpreting our experiments in terms of linear stability of small perturbations. The data indicate that, as expected, those experimental manipulations that stabilized microplasmodia around their initially round shape (i.e., Phalloidin treatment and Pluronic substrate coating) led to an increase in $\tau_{l o c}$. However, the manipulations that destabilized microplasmodia (i.e., LatA treatment and PolyL-lysine substrate coating) led to even more substantial increases in $\tau_{l o c}$, which is in apparent contradiction with the faster perturbation growth that one would expect in those conditions. This discrepancy brings up the limitations of linear stability analysis - predicting the long-term growth of shape perturbations usually requires full-blown simulations incorporating non-linear interfacial mechanics, substrate interactions, and mechano-chemical feedback. But it also highlights that experimental manipulations of biological systems usually have downstream effects on multiple parameters. For instance, weakening the F-actin cytoskeleton can cause a decrease in actomyosin contractile forces that drive cell shape changes [52]. Besides, increasing substrate adhesiveness can 

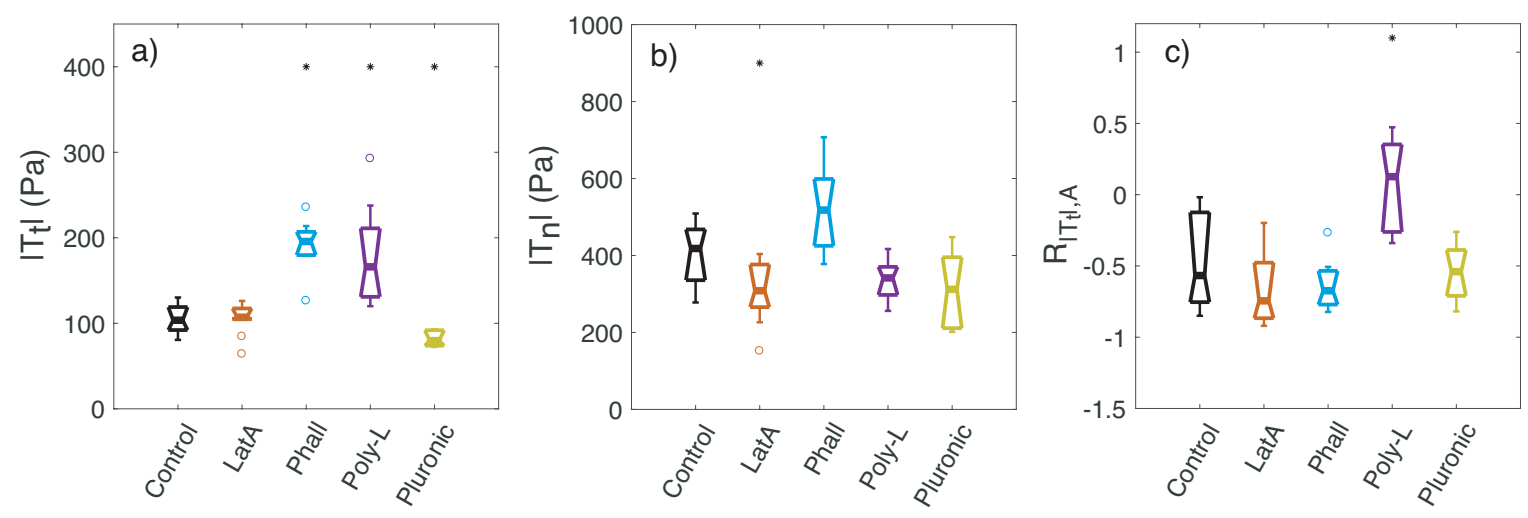

Figure 7: Cortical integrity and substrate adhesiveness affect the exertion of traction stresses. (a) Box plots of the mean magnitude of in-plane traction stresses $\left|\vec{T}_{t}\right|$ exerted by microplasmodia treated with latrunculin $\mathrm{A}(N=13)$ or phalloidin $(N=8)$, or seeded on substrates coated with poly-L-lysine $(\mathrm{N}=9)$ or pluronic $(\mathrm{N}=5)$, together with untreated microplasmodia seeded on collagen coated substrates (control, $N=8$ ). (b) Box plots of the mean out-of-plane traction stresses magnitude $\left|T_{n}\right|$ for the same microplasmodia in panel $(a)$. (c) Box plots of Spearman correlation coefficient between $\left|\vec{T}_{t}\right|$ and and microplasmodial spread area. The asterisks denote statistically significant differences with respect to control conditions $(p<0.05$ according to Wilcoxon's ranksum test).

increase membrane tension by increasing the area of contact between the plasmodium and the substrate, which causes membrane stretching [53].

To evaluate the effect of our experimental manipulations in Physarum microplasmodia, we measured the average magnitudes of the in-plane and out-of-plane traction stresses $\left(\left|\vec{T}_{t}\right|\right.$ and $\left|T_{n}\right|$ respectively) for LatA, phalloidin, Poly-L-Lysine and pluronic treatments, and for control conditions. We also determined the correlation between microplasmodial spread area and in-plane traction stress magnitude, $R_{\left|T_{t}\right|, A}$ under these different conditions. The latter is an interesting parameter because it provides information about the role of mechanical forces in driving microplasmodial shape oscillations. In our experiments, LatA treatment did not lead to significant changes in $\left|\vec{T}_{t}\right|$ but it caused a moderate decrease in $\left|T_{n}\right|$ compared to control, consistent with reduced contractility of a slightly weaker actomyosin cytoskeleton (Figure $7 a-$ b). This reduced contractility probably explains why $\tau_{l o c}$ increased in LatA treated microplasmodia (Figure 6b, inset). Phalloidin treatment caused strong increases in both $\left|\vec{T}_{t}\right|$ and $\left|T_{n}\right|$. While this increase is compatible with the intended manipulation in cortical integrity, the resulting higher contractility may explain why $\tau_{l o c}$ only grew moderately with this manipulation (Figure 6b, inset). Substrate coating with pluronic did not significant affect $\left|T_{n}\right|$ or $R_{\left|T_{t}\right|, A}$ (Figure $7 b, c$ ) but it significantly decreased $\left|T_{t}\right|$ (Figure $7 a$ ), which is to be expected given that this treatment decreases substrate friction. Finally, substrate coating with poly-L-lysine significantly increased $\left|T_{t}\right|$ without significantly increasing $\left|T_{n}\right|$ (Figure $7 a, b$ ), consistent with the intended effect of the manipulation - to increase substrate friction. However, this coating also resulted in 
an almost total loss of correlation between $\left|T_{t}\right|$ and spread area (Figure $7 c$ ), implying that the increased friction made it harder for the microplasmodium to change its shape by means of contractile stresses. This result may explain the dramatic increase in $\tau_{l o c}$ observed for poly-L-lysine (Figure $6 c$, inset), even if this manipulation overall favored symmetry breaking at smaller microplasmodial radii.
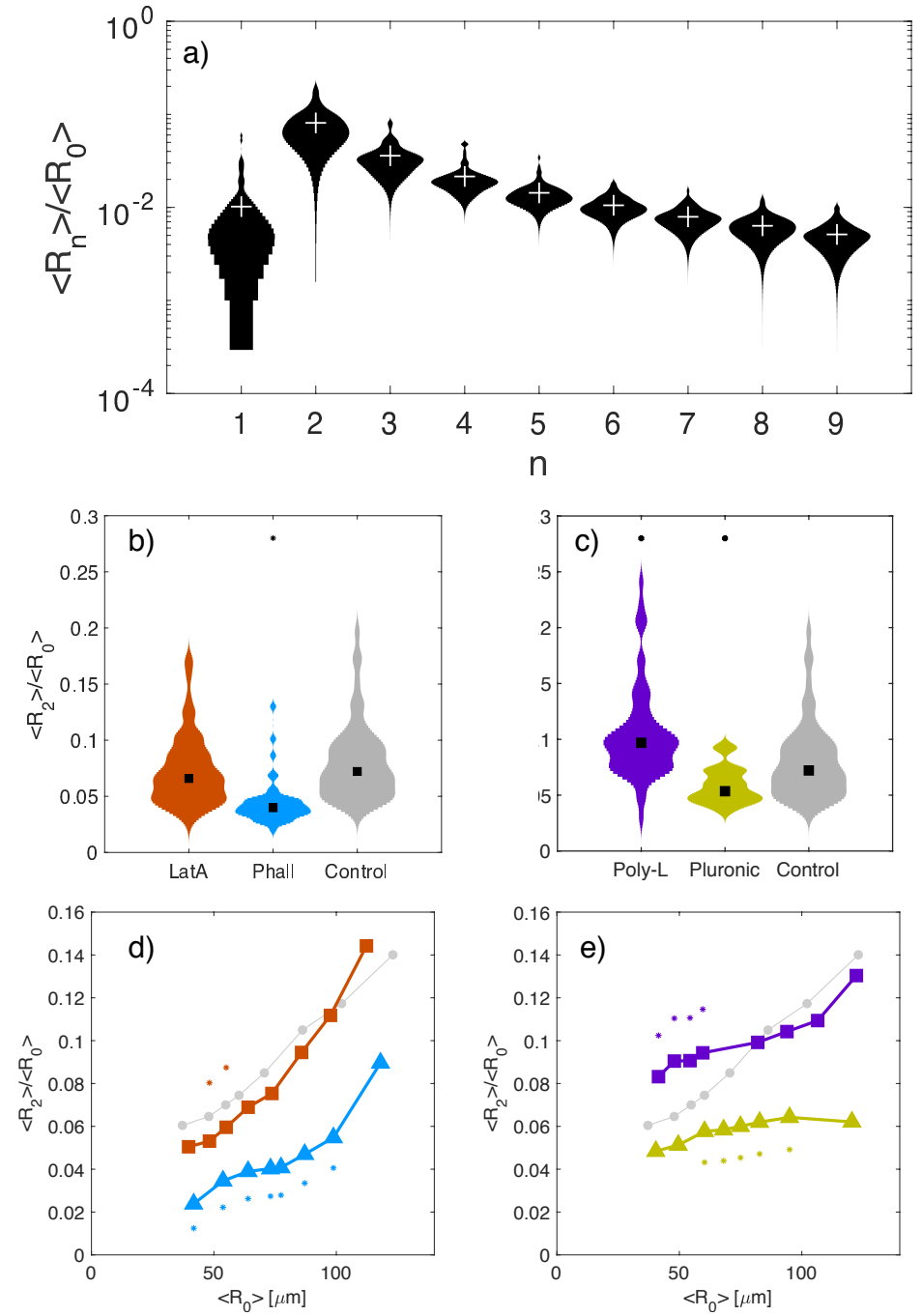

Figure 8: Size, cortical integrity and substrate adhesiveness determine the morphology of fragments. (a) Distributions of normalized shape mode amplitudes $\left\langle R_{n}\right\rangle /\left\langle R_{0}\right\rangle$ vs. lobe number. (b) Normalized amplitude of the dominant shape mode, $\left\langle R_{2}\right\rangle /\left\langle R_{0}\right\rangle$ for microplasmodia treated with latrunculin $\mathrm{A}(N=70)$, phalloidin $(N=48)$ as well as untreated ones $(N=61)$. (c) $\left\langle R_{2}\right\rangle /\left\langle R_{0}\right\rangle$ for microplasmodia seeded on substrates coated with poly-L-lysine $(N=66)$, pluronic $(N=39)$ and collagen (control, $N=61)$. (d) $\left\langle R_{2}\right\rangle /\left\langle R_{0}\right\rangle$ vs. $\langle R\rangle$ for the three same cases as panel $(b)$. (e) $\left\langle R_{2}\right\rangle /\left\langle R_{0}\right\rangle$ vs. $\langle R\rangle$ for the three same cases as panel $(c)$. The asterisks denote statistically significant differences with respect to control conditions $(p<0.05$ according to Wilcoxon's ranksum test). 


\subsection{Dominant Shape Oscillation Modes}

As noted above, Physarum microplasmodia of all sizes underwent pronounced shape fluctuations upon seeding onto the substrate (see Figure 3). Figure 8(a) shows the distributions of shape modes as a function of lobe number obtained from the morphological analysis described in section 2.7, after normalizing with $R_{0}$ and averaging over time. The data indicate that the most dominant lobe number in Physarum microplasmodia that initiated locomotion was $n=2$, which in combination with the prominent albeit less dominant mode $n=1$ corresponds to an oval/tadpole-like shape (see Figure $1 a$ ). The second most dominant mode was $n=3$, which in combination with modes $n=2$ and $n=1$ yields a tadpole-like shape with two frontal pseudopods (see Figure $1 b$ ). Remarkably, lobe number $n=2$ was consistently dominant for $98 \%$ of the microplasmodia (281 out of 287) both in control conditions and in experiments where we manipulated the F-actin cytoskeleton and substrate adhesiveness. Together with the similar shapes of the Rate $_{L o c}$ vs. $R_{0}$ curves observed in Figure 6 for all conditions, this result suggests that all the microplasmodia in our experiments underwent a similar instability mechanism to initiate locomotion.

Figure $8(b)-(c)$ shows that the dominant shape perturbations became significantly weaker in Phalloidin-treated microplasmodia and in substrates coated with Pluronic, whereas they became significantly stronger in Poly-L-lysine coated substrates. These results provide additional evidence suggesting that the instability driving the onset of locomotion is strengthened by substrate adhesiveness and weakened by cortical stiffness. Furthermore, Figure $8(d)-(e)$ indicates that the amplitude of the dominant shape perturbations increased with $\left\langle R_{0}\right\rangle$, particularly beyond values approximately equal to the critical radius of initiation of locomotion observed in Figure 6. Microplasmodia seeded onto Pluronic-treated substrates deviated from this behavior, suggesting that larger size may not confer an important advantage for locomotion in less adhesive substrates.

\section{Conclusion}

The plasmodium Physarum polycephalum, a true slime mold, has been widely used as a model organism in studies of flow-driven amoeboid locomotion [1,2,12]. The plasmodium is a single cell with multiple nuclei and is composed of a gel-like submembranous ectoplasmic layer and a sol-like inner endoplasm. The endoplasm exhibits streaming flows of alternating direction induced by oscillatory contractions of the ectoplasm, which are in turn governed by the flow-mediated transport of chemical signals such as calcium ions $[7,16,20]$. A remarkable feature of this kind of flow-contraction coupling is that it generates long-range self-organization [54]. Consequently, the size of Physarum plasmodia can vary over several orders of magnitude without drastic qualitative changes in their overall behavior $[6,9]$. In contrast, their locomotor dynamics can be rather sensitive to plasmodial size. Particularly, Physarum microplasmodia of sizes smaller 
than $\sim 80$ microns do not migrate directionally whereas microplasmodia of a few hundred microns undergo rapid amoeboid locomotion $[7,9,16]$.

The transition between the non-migratory and the migratory states of Physarum microplasmodia is reminiscent of a symmetry breaking process $[7,8]$. Given the biological simplicity of Physarum and its very loose control over specimen size, this organism constitutes an excellent model to study symmetry breaking phenomena in biological active matter. More specifically, experiments with small Physarum microplasmodia of sizes ranging from tens to a few hundred microns allow for studying flow-driven amoeboid motility without the posterior vein structures usually found in fragments of larger size (e.g., mesoplasmodia [21]). However, the mechanics of symmetry breaking and the onset of locomotion in Physarum microplasmodia had not been studied experimentally before.

This study has revealed that substrate adhesiveness, microplasmodial size, and cortical strength govern the symmetry breaking and onset of locomotion of Physarum microplasmodia. Overall, our results are consistent with two not mutually exclusive views of the initiation of locomotion - one that idealizes the process as the instability of a two-phase active poroelastic material [14,15,44], and an alternate view that models symmetry breaking as an interfacial instability $[48,49]$. In both views, the integrity of the ectoplasm hinders the initiation of locomotion at short length scales, although by different physical mechanisms. In both scenarios, increased substrate friction favors the onset of locomotion of small fragments that would otherwise remain round and stable, consistent with our experiments on pluronic- and poly-L-lysine-coated substrates. However, we note that increased substrate friction also caused an increase in the experimentally observed timescale of symmetry breaking, suggesting that indefinitely increasing substrate adhesion should arrest Physarum locomotion, consistent with our previous observations [16].

Our spectral analysis of microplasmodial shape during symmetry breaking revealed that the onset of locomotion coincides with a transition from a round shape to a tadpole-like shape with two lobes or, albeit less frequently, three lobes. This result was not sensitive to manipulations in ectoplasm integrity or substrate adhesiveness, suggesting that the underlying mechano-chemical instability was the same for different microplasmodial sizes and experimental manipulations. Our measurements also revealed that an intense polarization of the mechanical properties of the ectoplasm accompanied the onset of locomotion - over $\sim 3$ hours, the elastic shear modulus of the posterior ectoplasm increased approximately three-fold, whereas its viscous modulus increased only by $\sim 50 \%$. The robustness of the mechano-chemical instability triggering directional locomotion and the anteroposterior polarization of ectoplasmic mechanical properties may facilitate the emergence of mechano-chemical wave patterns leading to directional locomotion, as previously speculated by $[10,11]$. Consistent with these ideas, disorganized mechano-chemical waves are a seldom occurrence in directionally migrating Physarum microplasmodia [20]. However, the present data could not clarify the previously reported emergence of different organized wave patterns upon symmetry 
breaking - i.e., traveling waves (peristaltic locomotion) and standing waves (amphistaltic locomotion) $[7,16,19,20]$. Given that peristaltic locomotion is significantly faster than amphistaltic locomotion, this question warrants future investigation.

In summary, the present study suggests that a mechano-chemical instability governs the onset of locomotion in Physarum microplasmodia. The nature of the instability does not seem to depend on substrate adhesiveness, microplasmodial size, or cortex integrity; however, the observed growth rates are sensitive to these factors. Specifically, the initiation of locomotion takes place when microplasmodial size is larger than a critical value, and this critical size decreases with increasing substrate adhesiveness or weakening cortex integrity. The posterior cortex becomes significantly stiffer concurrent to this process, which presumably favors directional locomotion by preventing the growth of shape fluctuations at the tail of the microplasmodium. While these experimental measurements leave open questions about how mechanical factors work together to enable flow-driven amoeboid locomotion, they also provide original quantitative data with an unprecedented level of detail that can be used to inspire and validate future modeling studies aimed at addressing those questions. 


\section{References}

[1] Allen RD, Allen NS. Cytoplasmic streaming in amoeboid movement. Annual review of biophysics and bioengineering. 1978;7(1):469-495.

[2] Gawlitta W, Wolf KV, Hoffmann HU, Stockem W. Studies on Microplasmodia of Physarum polycephalum. I. Classification and Locomotion Behavior. Cell and tissue research. 1980;209(1):71-86.

[3] Nakagaki T, Yamada H, Tóth Á. Intelligence: Maze-solving by an amoeboid organism. Nature. 2000;407(6803):470.

[4] Piovanelli M, Fujie T, Mazzolai B, Beccai L. A Bio-Inspired Approach Towards the Development of Soft Amoeboid Microrobots. In: Biomedical Robotics and Biomechatronics, the IEEE RAS EMBS International Conference on. IEEE; 2012. p. 612-616.

[5] Kamiya N, Allen RD, Yoshimoto Y. Dynamic organization of Physarum plasmodium. Cell Motility;10(1-2):107-116. Available from: https://onlinelibrary.wiley.com/doi/abs/10. $1002 / \mathrm{cm} .970100115$.

[6] Daniel JW, Rusch HP. The Pure Culture of Physarum polycephalum on a Partially Defined Soluble Medium. Microbiology. 1961;25(1):47-59. Available from: https://mic. microbiologyresearch.org/content/journal/micro/10.1099/00221287-25-1-47.

[7] Matsumoto K, Takagi S, Nakagaki T. Locomotive Mechanism of Physarum Plasmodia Based on Spatiotemporal Analysis of Protoplasmic Streaming. Biophysical Journal. 2008 Apr;94(7):24922504.

[8] Teplov VA. Role of mechanics in the appearance of oscillatory instability and standing waves of the mechanochemical activity in thePhysarum polycephalumplasmodium. Journal of Physics D: Applied Physics. 2017 may;50(21):213002. Available from: https://doi.org/10.1088\% 2F1361-6463\%2Faa6727.

[9] Koya S, Ueda T. The Onset of Rhythmic Streaming in the Physarum Plasmodium. ACH, models in chemistry. 1998;135(3):297-304.

[10] Ueda T, Matsumoto K, Akitaya T, Kobatake Y. Spatial and temporal organization of intracellular adenine nucleotides and cyclic nucleotides in relation to rhythmic motility in Physarum plasmodium. Experimental Cell Research. 1986;162(2):486 - 494. Available from: http: //www.sciencedirect.com/science/article/pii/0014482786903526.

[11] Takagi S, Ueda T. Emergence and transitions of dynamic patterns of thickness oscillation of the plasmodium of the true slime mold Physarum polycephalum. Physica D: Nonlinear Phenomena. 2008;237(3):420 - 427 .

[12] Kobayashi R, Tero A, Nakagaki T. Mathematical Model for Rhythmic Protoplasmic Movement in the True Slime Mold. Journal of Mathematical Biology. 2006 Aug;53(2):273-286. Available from: https://doi.org/10.1007/s00285-006-0007-0.

[13] Radszuweit M, Alonso S, Engel H, Bär M. Intracellular Mechanochemical Waves in an Active Poroelastic Model. Physical Review Letters. 2013;110(13):138102.

[14] Radszuweit M, Engel H, Bär M. An Active Poroelastic Model for Mechanochemical Patterns in Protoplasmic Droplets of Physarum polycephalum. PLoS ONE. 2014;9(6):e99220.

[15] Kulawiak DA, Löber J, Bär M, Engel H. Oscillatory motion of a droplet in an active poroelastic two-phase model. Journal of Physics D: Applied Physics. 2018 oct;52(1):014004. Available from: https://doi.org/10.1088\%2F1361-6463\%2Faae41d.

[16] Lewis OL, Zhang S, Guy RD, del Álamo JC. Coordination of contractility, adhesion and flow in migrating Physarum amoebae. Journal of The Royal Society Interface. 2015;12(106).

[17] Mondésert-Deveraux S, Allena R, Aubry D. A coupled friction-poroelasticity model of chimneying shows that confined cells can mechanically migrate without adhesions. 2018; .

[18] Rodiek B, Takagi S, Ueda T, Hauser MJ. Patterns of cell thickness oscillations during directional migration of Physarum polycephalum. European Biophysics Journal. 2015;44(5):349-358.

[19] Rieu JP, Delanoë-Ayari H, Takagi S, Tanaka Y, Nakagaki T. Periodic traction in migrating large 
amoeba of Physarum polycephalum. Journal of The Royal Society Interface. 2015;12(106).

[20] Zhang S, Guy RD, Lasheras JC, del Alamo JC. Self-organized mechano-chemical dynamics in amoeboid locomotion of Physarum fragments. Journal of Physics D: Applied Physics. 2017;50(20):204004.

[21] Oettmeier C, Döbereiner HG. A lumped parameter model of endoplasm flow in Physarum polycephalum explains migration and polarization-induced asymmetry during the onset of locomotion. PloS one. 2019;14(4):e0215622.

[22] Del Alamo JC, Meili R, Alvarez-Gonzalez B, Alonso-Latorre B, Bastounis E, Firtel R, et al. ThreeDimensional Quantification of Cellular Traction Forces and Mechanosensing of Thin Substrata by Fourier Traction Force Microscopy. PLoS ONE. 2013 Sep;8(9):e69850.

[23] Wang YL, Pelham RJ. Preparation of a Flexible, Porous Polyacrylamide Substrate for Mechanical Studies of Cultured Cells. Methods in Enzymology. 1998;298:489-496.

[24] Tse JR, Engler AJ. Preparation of Hydrogel Substrates with Tunable Mechanical Properties. Current protocols in Cell Biology. 2010 Jun;Chapter 10:10.16.1-10.16.16.

[25] Alvarez-Gonzalez B, Zhang S, Meili R, Firtel RA, Lasheras JC, Del Alamo JC. Two-Layer Elastographic 3-D Traction Force Microscopy. Scientific Reports. 2017;7:39315.

[26] Edelstein A, Amodaj N, Hoover K, Vale R, Stuurman N. Computer Control of Microscopes Using Micro-Manager. Current Protocols in Molecular Biology. 2010 Oct;Chapter 14:14.20.1-14.20.17.

[27] Serrano R, Aung A, Yeh YT, Varghese S, Lasheras JC, del Álamo JC. Three-dimensional Monolayer Stress Microscopy. Biophysical Journal. 2019;In press.

[28] Del Alamo JC, Meili R, Alonso-Latorre B, Rodriguez-Rodriguez J, Aliseda A, Firtel RA, et al. Spatio-Temporal Analysis of Eukaryotic Cell Motility by Improved Force Cytometry. Proceedings of the National Academy of Sciences. 2007 Aug;104(33):13343-13348.

[29] Cooper JA. Effects of cytochalasin and phalloidin on actin. J Cell Biol. 1987;105(4):1473-1478.

[30] Coué M, Brenner SL, Spector I, Korn ED. Inhibition of actin polymerization by latrunculin A. FEBS Letters. 1987;213(2):316 - 318. Available from: http://www.sciencedirect.com/ science/article/pii/0014579387815132.

[31] Bridgett MJ, Davies MC, Denyer SP. Control of staphylococcal adhesion to polystyrene surfaces by polymer surface modification with surfactants. Biomaterials. 1992;13(7):411 - 416. Available from: http://www.sciencedirect.com/science/article/pii/014296129290159L.

[32] Mazia D, Schatten G, Sale W. Adhesion of cells to surfaces coated with polylysine. Applications to electron microscopy. The Journal of Cell Biology. 1975;66(1):198-200. Available from: http://jcb.rupress.org/content/66/1/198.

[33] Gómez-González M, Del Alamo JC. Two-point particle tracking microrheology of nematic complex fluids. Soft matter. 2016;12(26):5758-5779.

[34] Del Alamo JC, Norwich GN, Li YsJ, Lasheras JC, Chien S. Anisotropic rheology and directional mechanotransduction in vascular endothelial cells. Proceedings of the National Academy of Sciences. 2008;105(40):15411-15416.

[35] Mason TG, Weitz D. Optical measurements of frequency-dependent linear viscoelastic moduli of complex fluids. Physical review letters. 1995;74(7):1250.

[36] Yamada S, Wirtz D, Kuo SC. Mechanics of Living Cells Measured by Laser Tracking Microrheology. Biophysical Journal. 2000 Apr;78(4):1736-1747.

[37] Mizuno D, Tardin C, Schmidt CF, MacKintosh FC. Nonequilibrium Mechanics of Active Cytoskeletal Networks. Science. 2007;315(5810):370-373. Available from: https://science. sciencemag. org/content/315/5810/370.

[38] Gupta SK, Guo M. Equilibrium and out-of-equilibrium mechanics of living mammalian cytoplasm. Journal of the Mechanics and Physics of Solids. 2017;107:284-293.

[39] Guy RD, Nakagaki T, Wright GB. Flow-induced channel formation in the cytoplasm of motile cells. Phys Rev E. 2011 Jul;84:016310. Available from: https://link.aps.org/doi/10.1103/ PhysRevE. 84.016310.

[40] Alvarez-Gonzalez B, Meili R, Bastounis E, Firtel RA, Lasheras JC, Del Alamo JC. Three- 
Dimensional Balance of Cortical Tension and Axial Contractility Enables Fast Amoeboid Migration. Biophysical Journal. 2015 Feb;108(4):821-832.

[41] Ohl C, Stockem W. Distribution and Function of Myosin II as a Main Constituent of the Microfilament System in Physarum Polycephalum. European Journal of Protistology. 1995 May;31(2):208-222.

[42] Meili R, Alonso-Latorre B, Del Álamo JC, Firtel RA, Lasheras JC. Myosin II Is essential for the spatiotemporal organization of traction forces during cell motility. Molecular biology of the cell. 2010;21(3):405-417.

[43] Ishigami M. Dynamic aspects of the contractile system in Physarum Plasmodium: I. Changes in spatial organization of the cytoplasmic fibrils according to the contraction-relaxation cycle. Cell Motility;6(5):439-447. Available from: https://onlinelibrary.wiley.com/doi/abs/10. $1002 / \mathrm{cm} .970060502$.

[44] Alonso S, Strachauer U, Radszuweit M, Bär M, Hauser MJB. Oscillations and uniaxial mechanochemical waves in a model of an active poroelastic medium: Application to deformation patterns in protoplasmic droplets of Physarum polycephalum. Physica D: Nonlinear Phenomena. 2016;318-319:58 - 69. Nonlinear Models in Molecular and Cell Biology.

[45] Kukulies J, Brix K, Stockem W. Studies on microplasmodia of Physarum polycephalum. Cell and Tissue Research. 1987 Oct;250(1):125-134. Available from: https://doi.org/10.1007/ BF00214663.

[46] Kukulies J, Stockem W. Function of the microfilament system in living cell fragments of Physarum polycephalum as revealed by microinjection of fluorescent analogs. Cell and tissue research. $1985 ; 242(2): 323-332$.

[47] Oettmeier C, Lee J, Döbereiner HG. Form follows function: ultrastructure of different morphotypes of Physarum polycephalum. Journal of Physics D: Applied Physics. 2018;51(13):134006.

[48] Callan-Jones A, Joanny JF, Prost J. Viscous-fingering-like instability of cell fragments. Physical review letters. 2008;100(25):258106.

[49] Nagilla A, Prabhakar R, Jadhav S. Linear stability of an active fluid interface. Physics of Fluids. 2018;30(2):022109. Available from: https://doi.org/10.1063/1.5012109.

[50] Stone HA. Dynamics of drop deformation and breakup in viscous fluids. Annual Review of Fluid Mechanics. 1994;26(1):65-102.

[51] Bastounis E, Meili R, Alvarez-Gonzalez B, Francois J, Del Alamo JC, Firtel RA, et al. Both Contractile Axial and Lateral Traction Force Dynamics Drive Amoeboid Cell Motility. Journal of Cell Biology. 2014 Mar;204(6):1045-1061.

[52] Kraning-Rush CM, Carey SP, Califano JP, Smith BN, Reinhart-King CA. The role of the cytoskeleton in cellular force generation in 2D and 3D environments. Physical biology. 2011;8(1):015009.

[53] Murrell MP, Voituriez R, Joanny JF, Nassoy P, Sykes C, Gardel ML. Liposome adhesion generates traction stress. Nature Physics. 2014;10(2):163.

[54] Julien JD, Alim K. Oscillatory fluid flow drives scaling of contraction wave with system size. Proceedings of the National Academy of Sciences. 2018;115(42):10612-10617. Available from: https://www. pnas.org/content/115/42/10612. 